\title{
Synchronization of Coupled Harmonic Oscillators Using Quantized Sampled Position Data
}

\author{
Xinjing Wang and Peipei He \\ The School of Resources and Environment, North China University of Water Resource and Electric Power, Zhengzhou 450045, China \\ Correspondence should be addressed to Xinjing Wang; wangxinjing888@163.com
}

Received 27 February 2017; Revised 25 April 2017; Accepted 29 May 2017; Published 3 July 2017

Academic Editor: Wenwu Yu

Copyright (C) 2017 Xinjing Wang and Peipei He. This is an open access article distributed under the Creative Commons Attribution License, which permits unrestricted use, distribution, and reproduction in any medium, provided the original work is properly cited.

\begin{abstract}
For coupled harmonic oscillators (CHO), the paper studies the synchronization problem by using the quantized current sampled position data. The logarithmic quantizer is adopted here to quantize the information transmitted; thus the quantization error of the sampled position data can be illustrated as the uncertainty according to the sector bound property. On the basis of that, the synchronization problem is converted into the asymptotical stability of the subsystems and even the solving problem of characteristic equation. Some sufficient conditions ensuring the synchronization of $\mathrm{CHO}$ are obtained relating to coupling strength, sampling period, and quantizer parameter. The usefulness of the theoretical result is shown by an example at the end.
\end{abstract}

\section{Introduction}

Recently, as a special multiagent system, $\mathrm{CHO}$ has gotten much attention. By assuming that the undirected network is not connectivity, literature [1] shows that the $\mathrm{CHO}$ is also able to achieve synchronization. When the $\mathrm{CHO}$ is affected by controller loss and communication time-delays, an algorithm is proposed in [2] to ensure the synchronization of the system and a brief procedure of the convergence analysis for such algorithm is given. By using some special analysis tools, literature [3] shows that the $\mathrm{CHO}$ can be synchronized under the conditions of mild connectivity for the directed graph. A frequency dependent topology condition is given in [4] to synchronize the $\mathrm{CHO}$. With the measurement noise, literature [5] gives an effective algorithm and the convergence analysis of the $\mathrm{CHO}$ for the directed network topologies with or without leaders. A sufficient condition for the synchronized oscillatory motions of $\mathrm{CHO}$ is given by the influence of noise for the strongly connected directed graph.

The control algorithms proposed in the above literature use both velocity information and position information. In some cases, each oscillator exchanges just the velocity information or the position information with its neighbors. The relating papers which design control algorithms just use velocity information or position information include [6-9].
Assuming that the network is connected, the sufficient conditions are given for the synchronized oscillatory motions by just using velocity information in brief [6]. The results of [6] are generalized to the sampled-data $\mathrm{CHO}$ affected by input signals loss in [7]. Literature [8] proposes a distributed control method just using the outdated position states. For both positive and negative coupling strengths, it derives some necessary and sufficient conditions (NSCs) for the synchronization of CHO. In [9], by adopting current and outdated position data, respectively, two distributed protocols are adopted for the synchronization of $\mathrm{CHO}$.

On the other hand, as an important issue, data quantization has received extensive attention in multiagent systems. Theoretical results in the past two years include [10-16]. The consensus of multiagent systems is studied according to the robust learning control approach in [10]. For the undirected connected graphs, literature [11] obtains some strong results for the problem of expected time to convergence for quantized consensus. Under the influence of quantized input and disturbances, the distributed quantized $H_{\infty}$ consensus is investigated in [12]. For the high-order multiagent systems, the quantized consensus is studied in [13], in which it assumed that only the first state can be measured. By adopting both uniform and logarithmic quantizers, some quantized consensus results are achieved 
for heterogeneous systems in [14]. By using probabilistic versus deterministic quantizers, the quantized consensus problem for multiagent systems is studied in [15]. Moreover, event-triggered consensus [16], averaging consensus [17], consensus tracking [18], metropolis consensus [19], containment control [20], and other consensus problems [2123] affected by data quantization are discussed in recent papers.

Summarized above, the synchronization of $\mathrm{CHO}$ and quantized consensus for the general multiagent systems have received enough attention, respectively. However, there are few results relating to the synchronization of $\mathrm{CHO}$ affected by data quantization, which is the problem studied in our paper. In fact, this paper's main contribution is to extend the results of literature [9] to the synchronization problem for $\mathrm{CHO}$ by using the quantized position data. By adopting the logarithmic quantizer, the quantization error of the sampled position data can be illustrated as the uncertainty according to the sector bound property. With this setup, some sufficient conditions relating to coupling strength, sampling period, and quantizer parameter are obtained for synchronized $\mathrm{CHO}$.

The organizational structure of the paper is as follows. Section 2 demonstrates the problem discussed and the $\mathrm{CHO}$ network; distributed protocol and logarithmic quantizer are illustrated there in detail. Some sufficient conditions for synchronization of $\mathrm{CHO}$ and the detailed proving process are shown in Section 3. The usefulness of the theoretical results is illustrated by a simulation example in Section 4 . Section 5 gives some conclusions.

Notation. The $n$-dimensional Euclidean space is denoted by $\mathbb{R}^{n} . \mathbb{N}\left(\mathbb{R}^{+}\right)$indicates the positive integers (real numbers) set. We denote by $|c|$ the module of complex number $c . A^{-1}$ and $A^{T}$ are the inverse and the transpose of matrix $A$, respectively. $\mathbf{0}_{n}$ and $1_{n}$ denote the vectors with dimension $n \times 1$ whose elements are all zeros or ones, respectively. For a multiagent system, especially for $\mathrm{CHO}$, information exchange between agents can be modeled by a network or graph [24]. Let graph $\mathscr{G}$ be with node set $\Omega=\{1, \ldots, M\}$ and edge set $\Upsilon \subseteq \Omega \times \Omega$. If there exists at least one node having a directed path to every other node, the digraph $\mathscr{G}$ is called containing a directed spanning tree (DST). The adjacency matrix $\mathscr{A}=\left(a_{i j}\right)_{M \times M}$ satisfies $a_{i j}>0$ if $(j, i) \in \Upsilon$ and $a_{i j}=0$ if $(j, i) \notin \Upsilon$. $\mathscr{M}_{i}=\left\{j \mid a_{i j}>0, j \notin i\right\}, \forall i \in\{1, \ldots, M\}$ is a set including all the neighbor nodes of node $i$. We use $L=\left(l_{i j}\right)_{M \times M}$ to denote the Laplacian matrix, whose elements satisfy $l_{i j}=-a_{i j}(i \neq j)$ and $l_{i i}=\sum_{k=1, k \neq i}^{M} a_{i k}[25] .0=\left|\eta_{1}\right| \leq\left|\eta_{2}\right| \leq \cdots \leq\left|\eta_{M}\right|$ represent the eigenvalues of $L$ when the digraph $\mathscr{G}$ contains a DST. $\operatorname{diag}\left(a_{1}, \ldots, a_{M}\right)$ indicates the diagonal matrix with diagonal elements $a_{1}, \ldots, a_{M}$.

\section{Problem Formulation}

For continuous-time $\mathrm{CHO}$, we illustrate the synchronization problem and protocol by using the quantized sampled position data in this section.
For a positive constant $h$, the synchronization problem of the following $\mathrm{CHO}$ network composed by $M$ nodes is discussed:

$$
\begin{aligned}
& \dot{x}_{i}(t)=v_{i}(t), \\
& \dot{v}_{i}(t)=-h x_{i}(t)+u_{i}(t), \quad i \in\{1, \ldots, M\}
\end{aligned}
$$

with position states $x_{i}(t)$, velocity states $v_{i}(t)$, and control input $u_{i}(t)$ for node $i$.

Assuming that the information between nodes is transmitted over the network, then it must be quantized before transmission. A protocol using the quantized value of the current relative sampled position data is adopted in this paper:

$$
\begin{aligned}
u_{i}(t)=\gamma \sum_{j \in \mathscr{M}_{i}} a_{i j}\left(q\left(x_{i}\left(t_{k}\right)\right)-q\left(x_{j}\left(t_{k}\right)\right)\right), \\
\quad t \in\left[t_{k}, t_{k+1}\right), i \in\{1, \ldots, M\}, k \in \mathbb{N},
\end{aligned}
$$

in which coupling strength $\gamma>0$ is to be designed and $x_{i}\left(t_{k}\right), i \in\{1, \ldots, M\}$, denote the sampled position at time instants $t_{k}=k T, k \in \mathbb{N}$, with $t_{0}=0$. The quantizer used here is a general logarithmic one defined as $[26,27]$

$$
\begin{aligned}
& q(\chi) \\
& \quad= \begin{cases}\kappa_{w} & \text { if } \chi \in\left[\frac{1}{1+\delta} \kappa_{w}, \frac{1}{1-\delta} \kappa_{w}\right), \chi>0 \\
0 & \text { if } \chi=0 \\
-q(-\chi) & \text { if } \chi<0\end{cases}
\end{aligned}
$$

for any $\chi \in \mathbb{R}$, with $w \in \mathbb{N} \cup\{0\}, \delta \in(0,1), \kappa_{w+1}=\rho \kappa_{w}, v_{0}>$ 0 , and $\rho=(1-\delta) /(1+\delta) \in(0,1)$.

The aim of our paper is designing suitable $\gamma$ and $T$ such that the CHO (1) under the control input (2) achieves synchronization; that is,

$$
\begin{aligned}
& \lim _{t \rightarrow \infty}\left\|x_{i}(t)-x_{j}(t)\right\|=0, \\
& \lim _{t \rightarrow \infty}\left\|v_{i}(t)-v_{j}(t)\right\|=0
\end{aligned}
$$

for any $i, j \in\{1, \ldots, M\}(i \neq j)$.

\section{The Sufficient Conditions for Synchronization}

For CHO (1) with protocol (2), the synchronization problem is studied in this section. The sufficient conditions for synchronization are pursued on the basis of the following two lemmas.

Lemma 1 (see [28]). The fact that the graph $\mathscr{G}$ contains a DST is equal to the fact that the matrix $L$ has a simple zero eigenvalue and other eigenvalues having positive real parts. Furthermore, $1_{M}$ is the right eigenvector relating to zero eigenvalue of L. Moreover, we can always get a suitable $\phi$, which is left eigenvector relating to zero eigenvalue of $L$, satisfying $\phi^{T} L=0$ and $\phi^{T} 1_{M}=1$. 
Lemma 2 (see [29]). Let $p(s)=s^{2}+\left(\varrho_{1}+\mathbf{i} \sigma_{1}\right) s+\varpi_{0}+\mathbf{i} \sigma_{0}$ be a complex-coefficient polynomial with real constants $\Phi_{0}, \sigma_{0}, \emptyset_{1}$, and $\sigma_{1}$, and then the asymptotical stability of $p(s)$ is equivalent to $\Phi_{1}>0$ and ${\omega_{1}}_{1} \sigma_{1} \sigma_{0}+\varpi_{1}^{2} \Phi_{0}-\sigma_{0}^{2}>0$.

By using the above lemmas, the following theorem is given to discuss the synchronization of $\mathrm{CHO}$ (1) under protocol (2), which is the main result of this paper.

Theorem 3. If the graph $\mathscr{G}$ contains a DST and the initial states satisfy $\phi^{T} x(0)=\phi^{T} v(0)=0$ with $\phi$ defined in Lemma 1 , then network (1) under protocol (2) can achieve synchronization if $\gamma$ and $\mathrm{T}$ are selected satisfying

$$
\begin{aligned}
& \gamma \notin\left[\frac{h}{\max _{i \in\{2, \ldots, M\}} \operatorname{Re}\left(\widetilde{\xi}_{i}\right)}, \frac{h}{\min _{i \in\{2, \ldots, M\}} \operatorname{Re}\left(\bar{\xi}_{i}\right)}\right], \\
& T \notin\left\{\frac{l \pi}{\sqrt{h}} \mid l \in \mathbb{N}\right\}, \\
& \gamma<\frac{h \operatorname{Re}\left(\widetilde{\xi}_{i_{\max }}\right)}{\left|\widetilde{\xi}_{i_{\max }}\right|^{2}}, \\
& T \in \Gamma_{T}\left(\max _{i \in\{2, \ldots, M\}, l \in \mathbb{N}} \arctan \sqrt{e_{i l}}\right),
\end{aligned}
$$

where $\tilde{\xi}_{i}$ and $\bar{\xi}_{i}, i \in\{2, \ldots, M\}$ are, respectively, the nonzero eigenvalues of $L\left(I_{M}+\bar{\Lambda}\right)$ and $L\left(I_{M}-\bar{\Lambda}\right)$ with $\bar{\Lambda}=\delta I_{M}, i_{\max }=$ $\arg \max _{i \in\{2, \ldots, M\}}\left|\widetilde{\xi}_{i}\right|, \Gamma_{T}(\varphi) \triangleq\left\{T \mid \tan ^{2}(\sqrt{h} T / 2)>\tan ^{2} \varphi\right\}$, for any $\varphi \in[0, \pi / 2)$, and

$$
e_{i l}=\frac{\left|h-\gamma \xi_{i}\right|^{2} b_{i l}^{2}}{a_{i l} b_{i l}^{2}+a_{i l}^{2} c_{i l}}
$$

for any $i \in\{2, \ldots, M\}, l \in \mathbb{N}$ with $a_{i l}=h \gamma \operatorname{Re}\left(\xi_{i}^{l}\right)-\gamma^{2}\left|\xi_{i}^{l}\right|^{2}, \quad b_{i l}=$ $h \gamma \operatorname{Im}\left(\xi_{i}^{l}\right)$, and $c_{i l}=h^{2}-h \gamma \operatorname{Re}\left(\xi_{i}^{l}\right)$, in which $\xi_{i}^{l}$ denote possible values for $\xi_{i}\left(t_{k}\right), k \in \mathbb{N}$, defined by (10). Moreover, let $x(t)=$ $\left(x_{1}(t), \ldots, x_{M}(t)\right)^{T}$ and $v(t)=\left(v_{1}(t), \ldots, v_{M}(t)\right)^{T}$, and we obtain

$$
\begin{aligned}
\lim _{t \rightarrow \infty} x_{i}(t)= & \cos (\sqrt{h} t) \phi^{T} x(0) \\
& +\frac{1}{\sqrt{h}} \sin (\sqrt{h} t) \phi^{T} v(0)=0, \\
\lim _{t \rightarrow \infty} v_{i}(t)= & -\sqrt{h} \sin (\sqrt{h} t) \phi^{T} x(0) \\
& +\cos (\sqrt{h} t) \phi^{T} v(0)=0
\end{aligned}
$$

for all $i \in\{1, \ldots, M\}$.

Proof. By the property of logarithmic quantizer, we get $q\left(x_{i}\left(t_{k}\right)\right)=\left(1+\varepsilon_{i}\left(t_{k}\right)\right) x_{i}\left(t_{k}\right)$ with $\left|\varepsilon_{i}\left(t_{k}\right)\right| \leq \delta, \forall k \in \mathbb{N} \cup\{0\}, i \in$ $\{1, \ldots, M\}$. For any $t \in \mathbb{R}^{+} \cup\{0\}$, let $z_{i}(t)=\left(x_{i}(t), v_{i}(t)\right)^{T}$, $i \in\{1, \ldots, M\}$ and $z(t)=\left(z_{1}^{T}(t), \ldots, z_{M}^{T}(t)\right)^{T}$; then network (1) under protocol (2) can be rewritten as

$$
\begin{aligned}
& \dot{z}(t)=\left(I_{M} \otimes A\right) z(t)+\gamma(L \otimes B)\left[\begin{array}{c}
q\left(x_{1}\left(t_{k}\right)\right) \\
0 \\
q\left(x_{2}\left(t_{k}\right)\right) \\
0 \\
\vdots \\
q\left(x_{M}\left(t_{k}\right)\right) \\
0
\end{array}\right] \\
&=\left(I_{M} \otimes A\right) z(t)+\gamma(L \otimes B) \\
&+\left[\begin{array}{c}
1 \\
0 \\
\ddots
\end{array}\right]\left[\begin{array}{c}
\left(1+\varepsilon_{1}\left(t_{k}\right)\right) x_{1}\left(t_{k}\right) \\
\left(1+\varepsilon_{1}\left(t_{k}\right)\right) v_{1}\left(t_{k}\right) \\
\vdots \\
\left(1+\varepsilon_{M}\left(t_{k}\right)\right) x_{M}\left(t_{k}\right) \\
\left(1+\varepsilon_{M}\left(t_{k}\right)\right) v_{M}\left(t_{k}\right)
\end{array}\right] \\
&=\left(I_{M} \otimes A\right) z(t)+\gamma(L \otimes B)\left(I_{M} \otimes D\right) \\
& \cdot\left(\left(I_{M}+\Lambda\left(t_{k}\right)\right) \otimes I_{2}\right) z\left(t_{k}\right)=\left(I_{M} \otimes A\right) z(t) \\
&+ \gamma\left(L\left(I_{M}+\Lambda\left(t_{k}\right)\right) \otimes B\right) z\left(t_{k}\right), \\
& t \in\left[t_{k}, t_{k+1}\right), k \in \mathbb{N} \cup\{0\},
\end{aligned}
$$

where $A=\left[\begin{array}{cc}0 & 1 \\ -h & 0\end{array}\right], B=\left[\begin{array}{ll}0 & 0 \\ 1 & 0\end{array}\right], D=\left[\begin{array}{ll}1 & 0 \\ 0 & 0\end{array}\right]$, and $\Lambda\left(t_{k}\right)=$ $\operatorname{diag}\left\{\varepsilon_{1}\left(t_{k}\right), \ldots, \varepsilon_{M}\left(t_{k}\right)\right\}$.

For the matrix $L\left(I_{M}+\Lambda\left(t_{k}\right)\right), \forall k \in \mathbb{N} \cup\{0\}$, the following claim holds by Lemma 1 .

Claim. If the graph $\mathscr{G}$ contains a DST, then the matrix $L\left(I_{M}+\right.$ $\left.\Lambda\left(t_{k}\right)\right), \forall k \in \mathbb{N} \cup\{0\}$, has a simple zero eigenvalue and other eigenvalues having positive real parts. Furthermore, $\left[1 /\left(1+\varepsilon_{1}\left(t_{k}\right)\right), 1 /\left(1+\varepsilon_{1}\left(t_{k}\right)\right), \ldots, 1 /\left(1+\varepsilon_{M}\left(t_{k}\right)\right)\right]^{T}$ and $\phi$ defined in Lemma 1 are the right eigenvector and left eigenvector, respectively, relating to zero eigenvalue of $L\left(I_{M}+\Lambda\left(t_{k}\right)\right)$.

Based on the above claim, for any $k \in \mathbb{N} \cup\{0\}$, there must exist matrices $Q\left(t_{k}\right)=\left(p_{1}\left(t_{k}\right), \ldots, p_{M}\left(t_{k}\right)\right) \in \mathbb{R}^{M \times M}$ and $Q^{-1}\left(t_{k}\right)=\left(q_{1}\left(t_{k}\right), \ldots, q_{M}\left(t_{k}\right)\right)^{T} \in \mathbb{R}^{M \times M}$ with $p_{1}\left(t_{k}\right)=$ $\left[1 /\left(1+\varepsilon_{1}\left(t_{k}\right)\right), 1 /\left(1+\varepsilon_{1}\left(t_{k}\right)\right), \ldots, 1 /\left(1+\varepsilon_{M}\left(t_{k}\right)\right)\right]^{T}$ and $q_{1}\left(t_{k}\right)=$ $\phi$ such that

$$
\begin{aligned}
J\left(t_{k}\right) & =Q^{-1}\left(t_{k}\right) L\left(I_{M}+\Lambda\left(t_{k}\right)\right) Q\left(t_{k}\right) \\
& =\left(\begin{array}{cc}
0 & 0_{M-1}^{T} \\
0_{M-1} & \widehat{L}\left(t_{k}\right)
\end{array}\right)
\end{aligned}
$$


with Jordan canonical form $J\left(t_{k}\right)$ and block upper triangular matrix $\widehat{L}\left(t_{k}\right)$ represented as

$$
\widehat{L}\left(t_{k}\right)=\left(\begin{array}{ccccc}
\xi_{2}\left(t_{k}\right) & \tau & 0 & \cdots & 0 \\
0 & \xi_{3}\left(t_{k}\right) & \tau & \cdots & 0 \\
& & & \ddots & \\
0 & 0 & 0 & \cdots & \xi_{M}\left(t_{k}\right)
\end{array}\right)
$$

in which $\xi_{i}\left(t_{k}\right), i \in\{2, \ldots, M\}$ satisfying $\operatorname{Re}\left(\xi_{2}\left(t_{k}\right)\right) \leq \cdots \leq$ $\operatorname{Re}\left(\xi_{M}\left(t_{k}\right)\right)$ are the nonzero eigenvalues of $L\left(I_{M}+\Lambda\left(t_{k}\right)\right)$, and $\tau$ is equal to 1 or 0 .

Let $m(t)=\left(m_{1}^{T}(t), \ldots, m_{M}^{T}(t)\right)^{T}=\left(Q^{-1}\left(t_{k}\right) \otimes I_{2}\right) z(t), \forall t \in$ $\left[t_{k}, t_{k+1}\right)$ and $m_{i}(t) \in \mathbb{R}^{2}$, and then (8) gives that

$$
\begin{aligned}
\dot{m}(t) & =\left(Q^{-1}\left(t_{k}\right) \otimes I_{2}\right)\left(I_{M} \otimes A\right)\left(Q\left(t_{k}\right) \otimes I_{2}\right) m(t) \\
& +\gamma\left(Q^{-1}\left(t_{k}\right) \otimes I_{2}\right)\left(L\left(I_{M}+\Lambda\left(t_{k}\right)\right) \otimes B\right) \\
& +\left(Q\left(t_{k}\right) \otimes I_{2}\right) m\left(t_{k}\right)=\left(I_{M} \otimes A\right) m(t) \\
& +\gamma\left(J\left(t_{k}\right) \otimes B\right) m\left(t_{k}\right), \quad t \in\left[t_{k}, t_{k+1}\right), k \in \mathbb{N} \cup\{0\}
\end{aligned}
$$

indicating

$$
\begin{aligned}
\dot{m}_{1}(t)= & A m_{1}(t), \\
\dot{M}_{2}(t)= & \left(I_{M-1} \otimes A\right) M_{2}(t) \\
& +\gamma\left(\widehat{L}\left(t_{k}\right) \otimes B\right) M_{2}\left(t_{k}\right),
\end{aligned}
$$

for any $t \in\left[t_{k}, t_{k+1}\right), k \in \mathbb{N} \cup\{0\}$, with $M_{2}(t)=\left(m_{2}^{T}(t), \ldots\right.$, $\left.m_{M}^{T}(t)\right)^{T}$.

Next, we will verify that the asymptotic stability of subsystem (12b) is equivalent to the synchronization of (8) if the initial states satisfy $\phi^{T} x(0)=\phi^{T} v(0)=0$. On one hand, if $\lim _{t \rightarrow \infty} m_{i}(t)=\mathbf{0}_{2}, i \in\{2, \ldots, M\}$, combining with $z(t)=\left(Q\left(t_{k}\right) \otimes I_{2}\right) m(t), t \in\left[t_{k}, t_{k+1}\right)$ and $p_{1}\left(t_{k}\right)=[1 /(1+$ $\left.\left.\varepsilon_{1}\left(t_{k}\right)\right), 1 /\left(1+\varepsilon_{1}\left(t_{k}\right)\right), \ldots, 1 /\left(1+\varepsilon_{M}\left(t_{k}\right)\right)\right]^{T}$ gives $z_{i}(t) \rightarrow(1 /(1+$ $\left.\left.\varepsilon_{i}\left(t_{k}\right)\right)\right) m_{1}(t), t \in\left[t_{k}, t_{k+1}\right), i \in\{1, \ldots, M\}$. Moreover, by defining

$$
E(t)=\left[\begin{array}{cc}
\cos (\sqrt{h}(t)) & \frac{1}{\sqrt{h}} \sin (\sqrt{h}(t)) \\
-\sqrt{h} \sin (\sqrt{h}(t)) & \cos (\sqrt{h}(t))
\end{array}\right],
$$

the equalities $\dot{m}_{1}(t)=A m_{1}(t)$ and $\phi^{T} x(0)=\phi^{T} v(0)=0$ tell us that

$$
\begin{aligned}
m_{1}(t) & =e^{A t} m(0)=E(t)\left(\phi^{T} \otimes I_{2}\right) z(0) \\
= & {\left[\begin{array}{c}
\cos (\sqrt{h} t) \phi^{T} x(0)+\frac{1}{\sqrt{h}} \sin (\sqrt{h} t) \phi^{T} v(0) \\
-\sqrt{h} \sin (\sqrt{h} t) \phi^{T} x(0)+\cos (\sqrt{h} t) \phi^{T} v(0)
\end{array}\right] } \\
= & \mathbf{0}_{2}
\end{aligned}
$$

for any $t \in \mathbb{R}^{+} \cup\{0\}$. Thus we get $\lim _{t \rightarrow \infty} z_{i}(t)=\mathbf{0}_{2}, i \in$ $\{1, \ldots, M\}$ which means that the synchronization of (8) is guaranteed with synchronization state $\mathbf{0}_{2}$. On the other hand, if the synchronization of (8) is ensured, by letting the synchronization state as $z^{*}(t)=\left(x^{*}(t), v^{*}(t)\right)^{T} \in \mathbb{R}^{2}$, we will show $\left(x^{*}(t), v^{*}(t)\right)^{T}=\mathbf{0}_{2}$. In fact, it holds that $m_{1}(t)=$ $\left(\phi^{T} \otimes I_{2}\right) z(t)=\sum_{i=1}^{M} \phi_{i} z_{i}(t)$ according to $m(t)=\left(Q^{-1} \otimes I_{2}\right) z(t)$. Based on $\phi^{T} 1_{M}=1$, we get $z^{*}(t)=m_{1}(t)$. Combined with (14), it gives $x^{*}(t)=v^{*}(t)=0$, and thus $\lim _{t \rightarrow \infty} z(t)=$ $\mathbf{0}_{2 M}$. Taking $m(t)=\left(Q^{-1}\left(t_{k}\right) \otimes I_{2}\right) z(t), \forall t \in\left[t_{k}, t_{k+1}\right)$, into consideration, we get $\lim _{t \rightarrow \infty} m_{i}(t)=\mathbf{0}_{2}, i \in\{1, \ldots, M\}$, which ensures the asymptotic stability of subsystem (12b).

By the claim above and (10), we know that the asymptotic stability of (12b) is equal to that of the following subsystems:

$$
\dot{m}_{i}(t)=A m_{i}(t)+\gamma \xi_{i}\left(t_{k}\right) B m_{i}\left(t_{k}\right), \quad i \in\{2, \ldots, M\},
$$

the solution of which is as

$$
\begin{aligned}
m_{i}(t) & =e^{A\left(t-t_{k}\right)} m_{i}\left(t_{k}\right)+\gamma \xi_{i}\left(t_{k}\right) \int_{t_{k}}^{t} e^{A(t-s)} d s B m_{i}\left(t_{k}\right) \\
& =e^{A\left(t-t_{k}\right)} m_{i}\left(t_{k}\right)+\gamma \xi_{i}\left(t_{k}\right) \int_{0}^{t-t_{k}} e^{A s} d s B m_{i}\left(t_{k}\right) \\
& =E_{i}\left(t-t_{k}\right) m_{i}\left(t_{k}\right)+F_{i}\left(\xi_{i}\left(t_{k}\right), t-t_{k}\right) m_{i}\left(t_{k}\right)
\end{aligned}
$$

for any $t \in\left[t_{k}, t_{k+1}\right), k \in \mathbb{N} \cup\{0\}, i \in\{1, \ldots, M\}$, where

$$
\begin{aligned}
& E_{i}\left(t-t_{k}\right) \\
& \quad=\left(\begin{array}{cc}
\cos \left(\sqrt{h}\left(t-t_{k}\right)\right) & \frac{1}{\sqrt{h}} \sin \left(\sqrt{h}\left(t-t_{k}\right)\right) \\
-\sqrt{h} \sin \left(\sqrt{h}\left(t-t_{k}\right)\right) & \cos \left(\sqrt{h}\left(t-t_{k}\right)\right)
\end{array}\right) \\
& F_{i}\left(\xi_{i}\left(t_{k}\right), t-t_{k}\right) \\
& =\left(\begin{array}{cc}
\frac{\gamma \xi_{i}\left(t_{k}\right)\left(1-\cos \left(\sqrt{h}\left(t-t_{k}\right)\right)\right)}{h} & 0 \\
\frac{\gamma \xi_{i}\left(t_{k}\right) \sin \left(\sqrt{h}\left(t-t_{k}\right)\right)}{\sqrt{h}} & 0
\end{array}\right) .
\end{aligned}
$$

Let $S_{i}\left(\xi_{i}\left(t_{k}\right), t-t_{k}\right)=E_{i}\left(t-t_{k}\right)+F_{i}\left(\xi_{i}\left(t_{k}\right), t-t_{k}\right)$; then equality (16) gives

$$
\begin{array}{r}
m_{i}(t)=S_{i}\left(\xi_{i}\left(t_{k}\right), t-t_{k}\right) S_{i}\left(\xi_{i}\left(t_{k-1}\right), T\right) \\
\cdot S_{i}\left(\xi_{i}\left(t_{k-2}\right), T\right), \ldots, S_{i}\left(\xi_{i}(0), T\right) m_{i}(0), \\
i \in\{2, \ldots, M\}
\end{array}
$$

for any $t \in\left[t_{k}, t_{k+1}\right), k \in \mathbb{N} \cup\{0\}$.

The boundedness of $S_{i}\left(\xi_{i}\left(t_{k}\right), t-t_{k}\right)$ results in that $m_{i}(t)$ tends to $\mathbf{0}_{2}$ if $\rho\left(S_{i}\left(\xi_{i}\left(t_{k}\right), T\right)\right)<1, k \in \mathbb{N} \cup\{0\}, i \in\{2, \ldots, M\}$. Denoting the possible values for $\xi_{i}\left(t_{k}\right), k \in \mathbb{N} \cup\{0\}, i \in$ $\{2, \ldots, M\}$ by $\xi_{i}^{l}, l \in \mathbb{N}$, then $\rho\left(S_{i}\left(\xi_{i}\left(t_{k}\right), T\right)\right)<1$ is ensured by 
$\rho\left(S_{i}\left(\xi_{i}^{l}, T\right)\right)<1$ which is equal to the fact that the following equation's solutions satisfy $|\lambda|<1$ :

$$
\begin{aligned}
f_{i l}(\lambda, T)= & \operatorname{det}\left(\lambda I_{2}-S_{i}\left(\xi_{i}^{l}, T\right)\right) \\
= & \lambda^{2} \\
& -\left(2 \cos (\sqrt{h} T)+\frac{1-\cos (\sqrt{h} T)}{h} \gamma \xi_{i}^{l}\right) \lambda \\
& +\left(1+\frac{\cos (\sqrt{h} T)-1}{h} \gamma \xi_{i}^{l}\right)=0 .
\end{aligned}
$$

If we set $\lambda=(s+1) /(s-1)$, characteristic equation (19) is rewritten as

$$
\begin{aligned}
2(1 & -\cos (\sqrt{h} T))\left(1-\frac{\gamma \xi_{i}^{l}}{h}\right) s^{2} \\
& +2(1-\cos (\sqrt{h} T)) \frac{\gamma \xi_{i}^{l}}{h} s+2(1+\cos (\sqrt{h} T)) \\
& =0
\end{aligned}
$$

for any $i \in\{2, \ldots, M\}, l \in \mathbb{N}$. According to the property of bilinear transformation, $|\lambda|<1$ achieves if and only if $\operatorname{Re}(s)<0$. Moreover, if we define $\bar{\Lambda}=\delta I_{M}$ and denote the nonzero eigenvalues of $L\left(I_{M}+\bar{\Lambda}\right)$ and $L\left(I_{M}-\bar{\Lambda}\right)$ as $\widetilde{\xi}_{i}$ and $\bar{\xi}_{i}, i \in\{2, \ldots, M\}$, respectively, according to the relationship between the eigenvalues and the parameters $\varepsilon_{i}, i \in\{1, \ldots, M\}$, we get

$$
\min _{i \in\{2, \ldots, M\}} \operatorname{Re}\left(\bar{\xi}_{i}\right) \leq \operatorname{Re}\left(\xi_{i}^{l}\right) \leq \max _{i \in\{2, \ldots, M\}} \operatorname{Re}\left(\widetilde{\xi}_{i}\right)
$$

which ensures that $h \neq \gamma \xi_{i}^{l}, i \in\{2, \ldots, M\}, l \in \mathbb{N}$, if (5a) is satisfied. Combining with (5b) gives that equality (20) can be transferred into, $\forall i \in\{2, \ldots, M\}, l \in \mathbb{N}$,

$$
s^{2}+\frac{\gamma \xi_{i}^{l}}{h-\gamma \xi_{i}^{l}} s+\frac{1+\cos (\sqrt{h} T)}{1-\cos (\sqrt{h} T)} \frac{h}{h-\gamma \xi_{i}^{l}}=0
$$

Considering $(1+\cos (\sqrt{h} T)) /(1-\cos (\sqrt{h} T))>0$, Lemma 2 tells us that the roots of (22) located in the left-half plane are equal to

$$
\begin{array}{r}
\frac{a_{i l}}{\left|h-\gamma \xi_{i}^{l}\right|^{2}}>0, \\
\frac{a_{i l} b_{i l}^{2}+a_{i l}^{2} c_{i l}}{\left|h-\gamma \xi_{i}^{l}\right|^{2}}-\frac{1+\cos (\sqrt{h} T)}{1-\cos (\sqrt{h} T)} b_{i l}^{2}>0,
\end{array}
$$

where

$$
\begin{aligned}
a_{i l} & =h \gamma \operatorname{Re}\left(\xi_{i}^{l}\right)-\gamma^{2}\left|\xi_{i}^{l}\right|^{2}, \\
b_{i l} & =h \gamma \operatorname{Im}\left(\xi_{i}^{l}\right), \\
c_{i l} & =h^{2}-h \gamma \operatorname{Re}\left(\xi_{i}^{l}\right)
\end{aligned}
$$

for all $i \in\{2, \ldots, M\}, l \in \mathbb{N}$. Recalling (5a), we have that condition (23) is equivalent to

$$
\gamma<\frac{h \operatorname{Re}\left(\xi_{i}^{l}\right)}{\left|\xi_{i}^{l}\right|^{2}}, \quad i \in\{2, \ldots, M\}, \quad l \in \mathbb{N},
$$

which is guaranteed by

$$
\gamma<\min _{i \in\{2, \ldots, M\}, l \in \mathbb{N}}\left\{\frac{h \operatorname{Re}\left(\xi_{i}^{l}\right)}{\left|\xi_{i}^{l}\right|^{2}}\right\} .
$$

Based on the relationship between the eigenvalues and the parameters $\varepsilon_{i}, i \in\{1, \ldots, M\}$ and inequality (21), we know that

$$
\min _{i \in\{2, \ldots, M\}, l \in \mathbb{N}}\left\{\frac{h \operatorname{Re}\left(\xi_{i}^{l}\right)}{\left|\xi_{i}^{l}\right|^{2}}\right\}=\frac{h \operatorname{Re}\left(\widetilde{\xi}_{i_{\max }}\right)}{\left|\tilde{\xi}_{i_{\max }}\right|^{2}}
$$

with $i_{\max }=\arg \max _{i \in\{2, \ldots, M\}}\left|\tilde{\xi}_{i}\right|$.

Moreover, (26) gives $\gamma<h / \operatorname{Re}\left(\xi_{i}^{l}\right)$ indicating $a_{i l}>0$ and $c_{i l}>0$. By defining

$$
e_{i l}=\frac{\left|h-\gamma \xi_{i}^{l}\right|^{2} b_{i l}^{2}}{a_{i l} b_{i l}^{2}+a_{i l}^{2} c_{i l}}
$$

we get that (24) holds if

$$
T \in \Gamma_{T}\left(\max _{i \in\{2, \ldots, M\}, l \in \mathbb{N}} \arctan \sqrt{e_{i l}}\right)
$$

with $\Gamma_{T}(\varphi), \varphi \in[0, \pi / 2)$ defined by $\Gamma_{T}(\varphi)=\left\{T \mid \tan ^{2}(\sqrt{h} T /\right.$ 2) $\left.>\tan ^{2} \varphi\right\}$. Therefore, if (5a) and (5b) are satisfied, we obtain that the roots of (20) hold negative real part if $(5 c)$ and (5d) hold which means that $|\lambda|<1$, where $\lambda$ denotes the eigenvalues of $S_{i}\left(\xi_{i}^{l}, T\right)$. Thus $\rho\left(S_{i}\left(\xi_{i}^{l}, T\right)\right)<1$ is guaranteed which gives $\lim _{t \rightarrow \infty} m_{i}(t)=\mathbf{0}_{2}$ indicating $\lim _{t \rightarrow \infty} z(t)=\mathbf{0}_{2 M}$. Up to now, it shows that the synchronization of network (1) under protocol (2) is obtained when (5a), (5b), (5c), and (5d) are satisfied. This completes the proof.

Remark 4. In this paper, the constraint on the initial state, $\phi^{T} x(0)=\phi^{T} v(0)=0$, is a necessary condition to ensure the synchronization of (8) according to $z_{i}(t) \rightarrow(1 /(1+$ $\left.\left.\varepsilon_{i}\left(t_{k}\right)\right)\right) m_{1}(t), t \in\left[t_{k}, t_{k+1}\right), i \in\{1, \ldots, M\}$, in which $\varepsilon_{i}$ and $\varepsilon_{j},(i \neq j)$ are different from each other. How to relax the constraint on the initial state is one of our further research directions, which will need completely different proof line.

Remark 5. When we design parameters based on the above theorem, it is obvious that the difficulty lies in ascertaining the value of $\varphi=\max _{i \in\{2, \ldots, M\}, l \in \mathbb{N}} \arctan \sqrt{e_{i l}}$. In fact, it can always increase or decrease the value of $\varepsilon_{i}, i \in\{1, \ldots, M\}$ to study the proportional relationship between $\varepsilon_{i}$ and $e_{i l}$ and thus determine the value of $\varphi$ and the feasible interval for T. 
Remark 6. It is worth mentioning that the synchronization expression of $\mathrm{CHO}$ (1) under protocol (2) is the same as the one under protocol [9]

$$
\begin{aligned}
& u_{i}(t)=\gamma \sum_{l \in \mathscr{M}_{i}} a_{i l}\left(x_{i}\left(t_{k}\right)-x_{j}\left(t_{k}\right)\right), \\
& \quad t \in\left[t_{k}, t_{k+1}\right), i \in\{1, \ldots, M\}, k \in \mathbb{N},
\end{aligned}
$$

where the current relative sampled position data has not been quantized. This is due to the fact that $E(t)$ defined in (13), which determines the synchronization state, is not influenced by quantization.

\section{Simulation}

A simulation example is given in this section to show the usefulness of the result. The $\mathrm{CHO}$ network composed of four nodes and the adjacency matrix $\mathscr{A}$ is given as

$$
\mathscr{A}=\left[\begin{array}{llll}
0 & 1 & 0 & 1 \\
1 & 0 & 0 & 1 \\
1 & 1 & 0 & 0 \\
0 & 1 & 1 & 0
\end{array}\right] .
$$

Select parameters as $h=0.8, \delta=0.1$, and $\gamma$ satisfying

$$
\begin{aligned}
& \gamma=0.2 \\
& \notin\left[\frac{h}{\max _{i \in\{2, \ldots, M\}} \operatorname{Re}\left(\widetilde{\xi}_{i}\right)}, \frac{h}{\min _{i \in\{2, \ldots, M\}} \operatorname{Re}\left(\bar{\xi}_{i}\right)}\right] \\
&=[0.2424,0.3556], \\
& \gamma=0.2<\frac{h \operatorname{Re}\left(\widetilde{\xi}_{i_{\max }}\right)}{\left|\tilde{\xi}_{i_{\max }}\right|^{2}}=0.2424 .
\end{aligned}
$$

Based on $\varphi=\max _{i \in\{2, \ldots, M\}, l \in \mathbb{N}} \arctan \sqrt{e_{i l}}=0.6255$ and [9]

$$
\begin{aligned}
\Gamma_{T}(\varphi)= & \left\{T \mid \tan ^{2}\left(\frac{\sqrt{h} T}{2}\right)>\tan ^{2} \varphi\right\} \\
= & \left(\bigcup_{l=0}^{\infty}\left(\frac{2(\varsigma \pi+\varphi)}{\sqrt{h}}, \frac{(2 \varsigma+1) \pi}{\sqrt{h}}\right)\right) \\
& \cup\left(\bigcup_{l=1}^{\infty}\left(\frac{(2 \varsigma-1) \pi}{\sqrt{h}}\right), \frac{2(\varsigma \pi-\varphi)}{\sqrt{h}}\right)
\end{aligned}
$$

we know $T \in(1.3987,3.5124)$ by setting $\varsigma=0$. Selecting $T=3 \in \Gamma_{T}(\varphi)$ and the initial states as $z(0)=$ $[-1,0,2,0,-1,2,-1,-1]^{T}$ satisfying $\phi^{T} x(0)=\phi^{T} v(0)=0$ with $\phi=[5 / 21,7 / 21,3 / 21,6 / 21]^{T}$, the synchronization states can be shown in Figure 1, from which we see that the $\mathrm{CHO}$ network reaches synchronization. If the initial state is set as $z(0)=[1,0,2,0,-1,2,-1,1]^{T}$ which violates $\phi^{T} x(0)=$ $\phi^{T} v(0)=0$, Figure 2 shows that the synchronization errors do not tend to zeros, which means that the synchronization for $\mathrm{CHO}$ network is not reached.
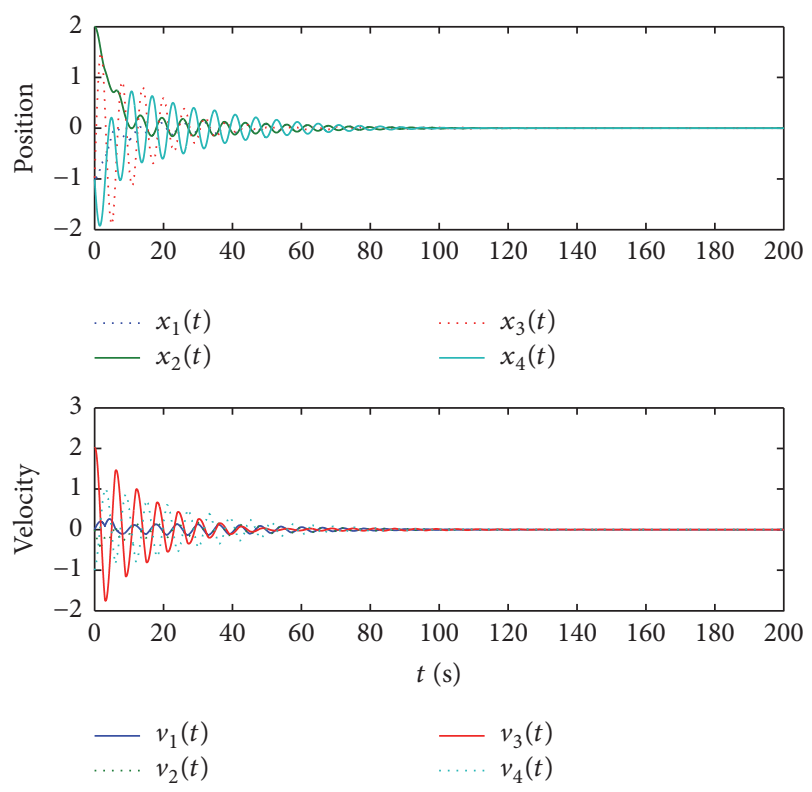

FIGURE 1: Synchronization with initial states $z(0)=[-1,0,2,0,-1,2$, $-1,-1]^{T}$.
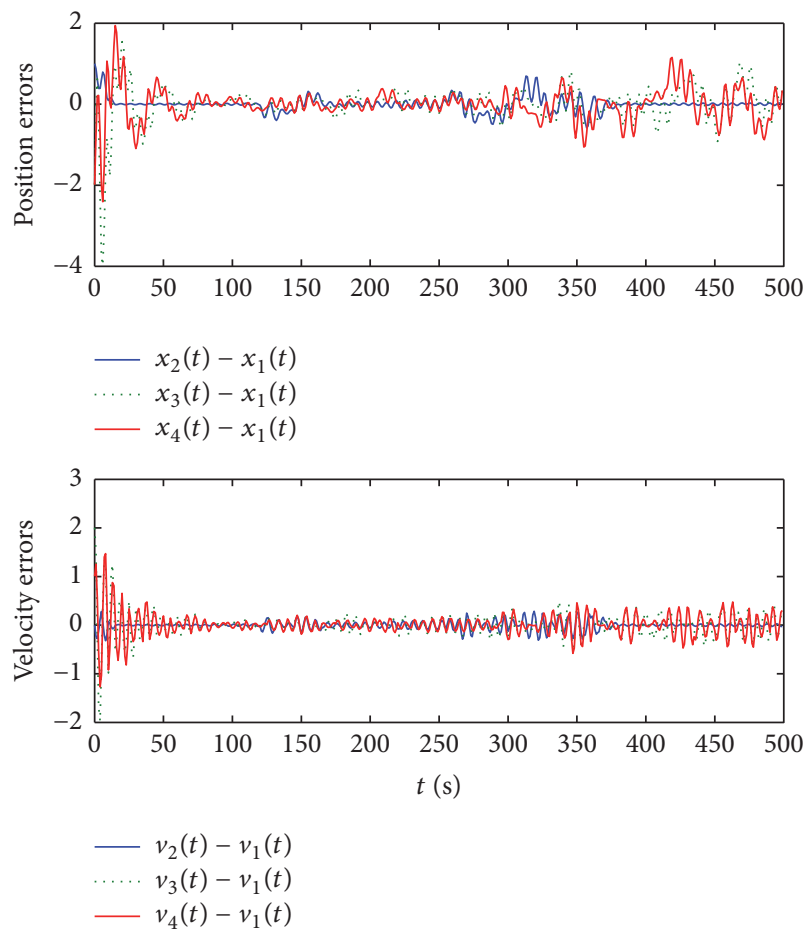

FIGURE 2: Synchronization errors with initial states $z(0)=[1,0,2,0$, $-1,2,-1,1]^{T}$.

\section{Conclusion}

By using the quantized current sampled position data, the sufficient conditions for synchronization of $\mathrm{CHO}$ are given according to coupling strength, sampling period, and quantizer parameter. It is worth mentioning that the results here need some restrictions on initial states. How to obtain the 
synchronization for $\mathrm{CHO}$ network without these restrictions holds some challenges, and it is one of our further research directions.

\section{Conflicts of Interest}

The authors declare that they have no conflicts of interest.

\section{Acknowledgments}

The work was supported by the National Natural Science Foundation of China (41501562, 61403125, and 61603108) and the State Key Laboratory of Coal Resources and Safe Mining (China University of Mining and Technology) (SKLCRSM16KFD02).

\section{References}

[1] H. Su, X. Wang, and Z. Lin, "Synchronization of coupled harmonic oscillators in a dynamic proximity network," Automatica, vol. 45, no. 10, pp. 2286-2291, 2009.

[2] J. Zhou, H. Zhang, L. Xiang, and Q. Wu, "Sampled-data synchronization of coupled harmonic oscillators with controller failure and communication delays," Theoretical and Applied Mechanics Letters, vol. 3, no. 6, Article ID 063002, 2013.

[3] W. Ren, "Synchronization of coupled harmonic oscillators with local interaction," Automatica, vol. 44, no. 12, pp. 3195-3200, 2008.

[4] X. Wang and Z. Cheng, "Synchronization of coupled discretetime harmonic oscillators with rational frequency," Institute of Electrical and Electronics Engineers. Transactions on Automatic Control, vol. 58, no. 6, pp. 1573-1579, 2013.

[5] J. Wang, J. Feng, C. Xu, M. Z. Q. Chen, Y. Zhao, and J. Feng, “The synchronization of instantaneously coupled harmonic oscillators using sampled data with measurement noise," Automatica, vol. 66, pp. 155-162, 2016.

[6] J. Zhou, H. Zhang, L. Xiang, and Q. Wu, "Synchronization of coupled harmonic oscillators with local instantaneous interaction," Automatica, vol. 48, no. 8, pp. 1715-1721, 2012.

[7] H. Zhang and J. Zhou, "Synchronization of sampled-data coupled harmonic oscillators with control inputs missing," Systems \& Control Letters, vol. 61, no. 12, pp. 1277-1285, 2012.

[8] Q. Song, W. Yu, J. Cao, and F. Liu, "Reaching Synchronization in Networked Harmonic Oscillators with Outdated Position Data," IEEE Transactions on Cybernetics, vol. 46, no. 7, pp. 1566-1578, 2016.

[9] Q. Song, F. Liu, G. Wen, J. Cao, and Y. Tang, "Synchronization of coupled harmonic oscillators via sampled position data control," IEEE Transactions on Circuits and Systems. I. Regular Papers, vol. 63, no. 7, pp. 1079-1088, 2016.

[10] T. Zhang and J. Li, "Robust iterative learning control of multiagent systems with logarithmic quantizer," in Proceedings of the 34th Chinese Control Conference, CCC 2015, pp. 7033-7038, chn, July 2015.

[11] S. R. Etesami and T. Başar, "Convergence Time for Unbiased Quantized Consensus over Static and Dynamic Networks," IEEE Transactions on Automatic Control, vol. 61, no. 2, pp. 443$455,2016$.

[12] X. Guo, J. Wang, F. Liao, and D. Wang, "Quantized Ho consensus of multi-Agent systems with quantization mismatch under switching weighted topologies," IEEE Transactions on Control of Network Systems, vol. PP, no. 99, 2015.

[13] Z. Qiu, L. Xie, and Y. Hong, "Quantized leaderless and leaderfollowing consensus of high-order multi-agent systems with limited data rate," Institute of Electrical and Electronics Engineers. Transactions on Automatic Control, vol. 61, no. 9, pp. 2432-2447, 2016.

[14] Y. Zhu, Y. Zheng, and L. Wang, "Quantised consensus of heterogeneous multi-agent systems," IET Control Theory \& Applications, vol. 9, no. 17, pp. 2553-2560, 2015.

[15] S. Zhu and B. Chen, "Quantized consensus by the ADMM: probabilistic versus deterministic quantizers," IEEE Transactions on Signal Processing, vol. 64, no. 7, pp. 1700-1713, 2016.

[16] Z. Zhang, L. Zhang, F. Hao, and L. Wang, "Periodic EventTriggered Consensus with Quantization," IEEE Transactions on Circuits and Systems II: Express Briefs, vol. 63, no. 4, pp. 406-410, 2016.

[17] M. El Chamie, J. Liu, and T. Basar, "Design and analysis of distributed averaging with quantized communication," Institute of Electrical and Electronics Engineers. Transactions on Automatic Control, vol. 61, no. 12, pp. 3870-3884, 2016.

[18] W. Xiong, X. Yu, Y. Chen, and J. Gao, "Quantized Iterative Learning Consensus Tracking of Digital Networks With Limited Information Communication," IEEE Transactions on Neural Networks and Learning Systems, 2016.

[19] T. Basar, S. R. Etesami, and A. Olshevsky, "Convergence time of quantized Metropolis consensus over time-varying networks," Institute of Electrical and Electronics Engineers. Transactions on Automatic Control, vol. 61, no. 12, pp. 4048-4054, 2016.

[20] X. Mu and K. Liu, "Containment control of single-integrator network with limited communication data rate," Institute of Electrical and Electronics Engineers. Transactions on Automatic Control, vol. 61, no. 8, pp. 2232-2238, 2016.

[21] L. Rong, H. Shen, and J. Li, "Distributed quantised consensus in groups of agents with acceleration-like inputs: a reference model-based scheme," IET Control Theory \& Applications, vol. 10, no. 5, pp. 590-598, 2016.

[22] Z. Zhang, L. Zhang, F. Hao, and L. Wang, "Leader-Following consensus for linear and lipschitz nonlinear multiagent systems with quantized communication," IEEE Transactions on Cybernetics, 2016.

[23] Z. Zeng, X. Wang, Z. Zheng, and L. Zhao, "Edge agreement of second-order multi-agent system with dynamic quantization via the directed edge Laplacian," Nonlinear Analysis. Hybrid Systems, vol. 23, pp. 1-10, 2017.

[24] H.-X. Hu, Q. Xuan, W. Yu, C.-G. Zhang, and G. Xie, "Secondorder consensus for heterogeneous multi-agent systems in the cooperation-competition network: a hybrid adaptive and pinning control approach," Nonlinear Analysis. Hybrid Systems, vol. 20, pp. 21-36, 2016.

[25] H.-X. Hu, W. Yu, G. Wen, Q. Xuan, and J. Cao, "Reverse group consensus of multi-agent systems in the cooperationcompetition network," IEEE Transactions on Circuits and Systems I: Regular Papers, vol. 63, no. 11, pp. 2036-2047, 2016.

[26] N. Elia and S. K. Mitter, "Stabilization of linear systems with limited information," Institute of Electrical and Electronics Engineers. Transactions on Automatic Control, vol. 46, no. 9, pp. 1384-1400, 2001.

[27] M. Fu and L. Xie, "The sector bound approach to quantized feedback control," Institute of Electrical and Electronics Engineers. Transactions on Automatic Control, vol. 50, no. 11, pp. 1698-1711, 2005. 
[28] W. Ren and R. W. Beard, "Consensus seeking in multiagent systems under dynamically changing interaction topologies," Institute of Electrical and Electronics Engineers. Transactions on Automatic Control, vol. 50, no. 5, pp. 655-661, 2005.

[29] P. C. Parks and V. Hahn, Stability Theory, Qrentice-Hall, NY, USA, 1993. 


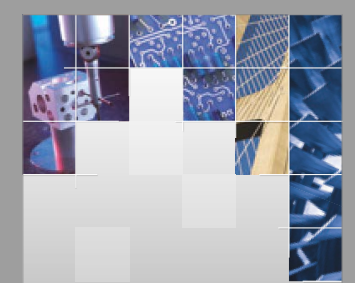

\section{Enfincering}
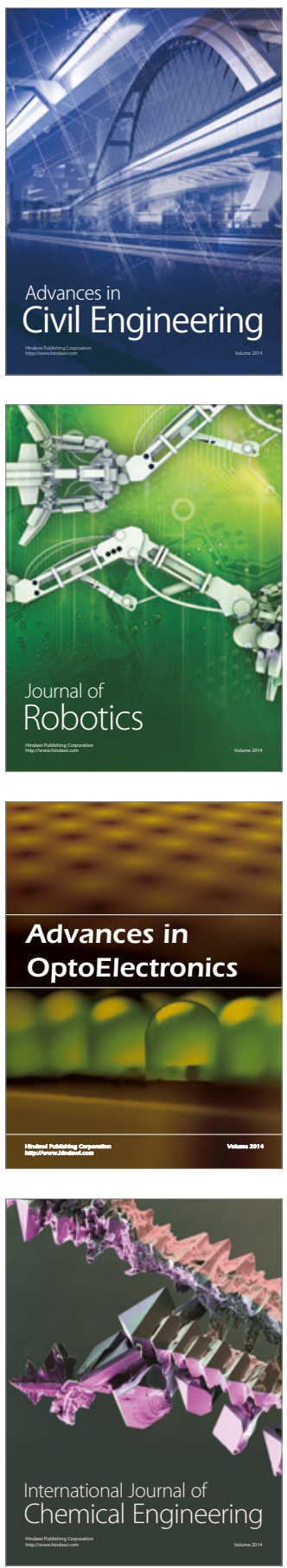

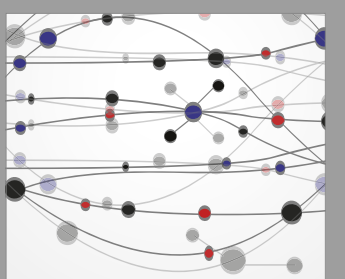

The Scientific World Journal

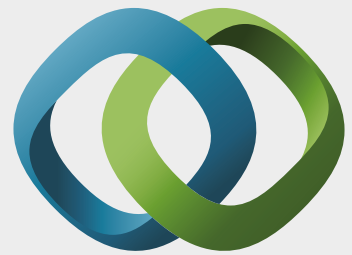

\section{Hindawi}

Submit your manuscripts at

https://www.hindawi.com
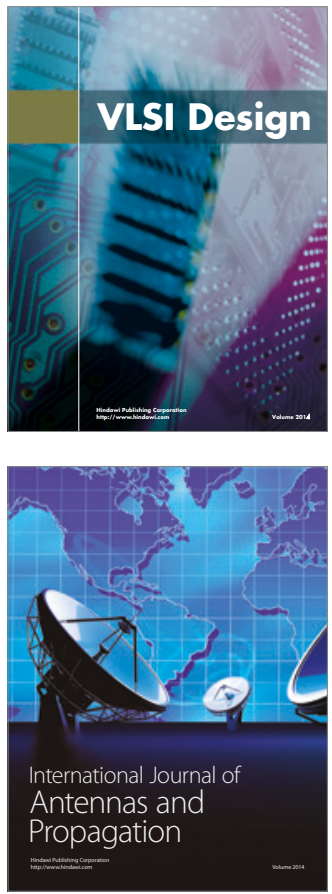

\section{Rotating}

Machinery
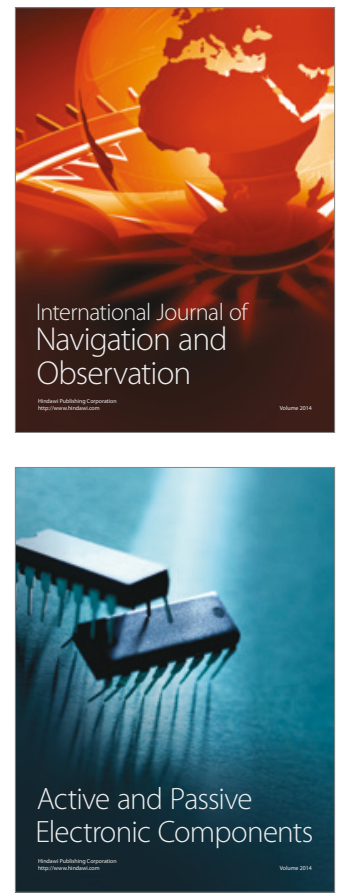
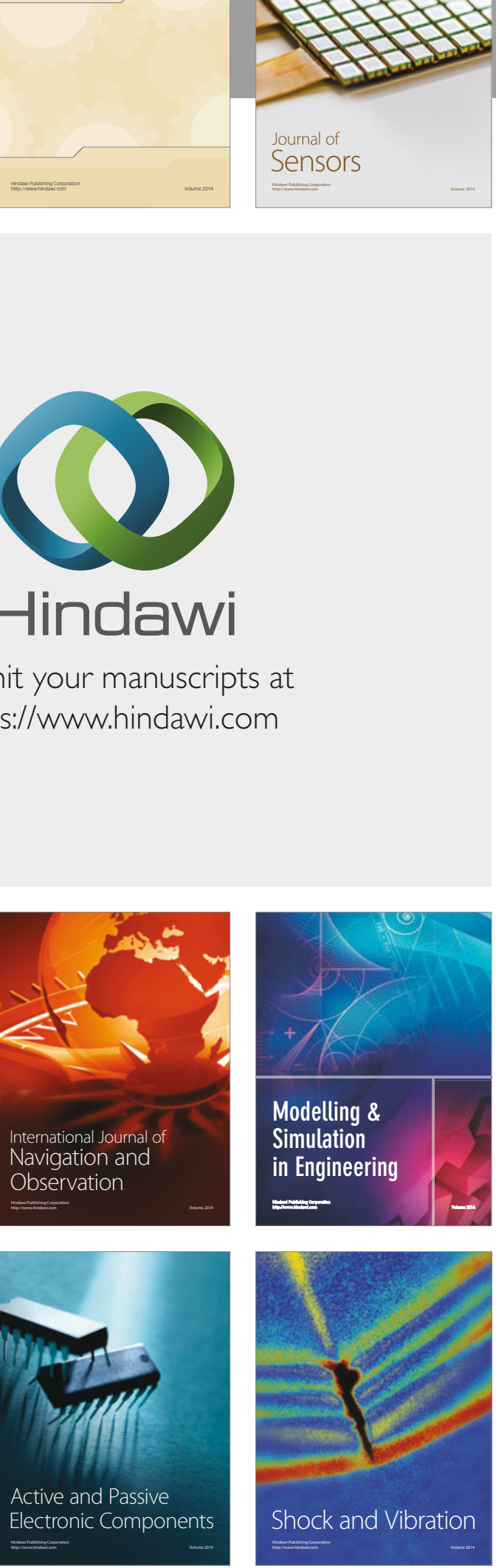
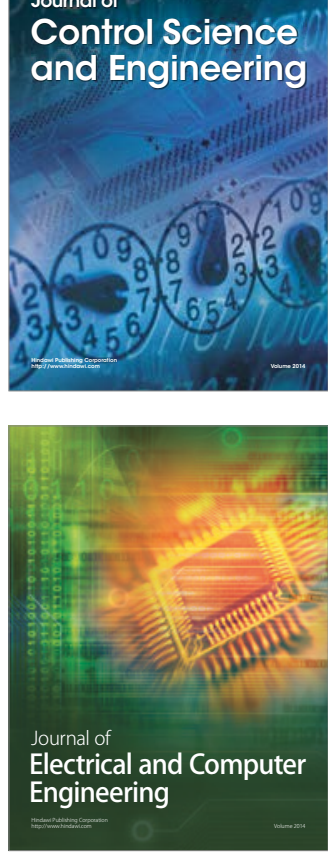

Distributed

Journal of

Control Science

and Engineering
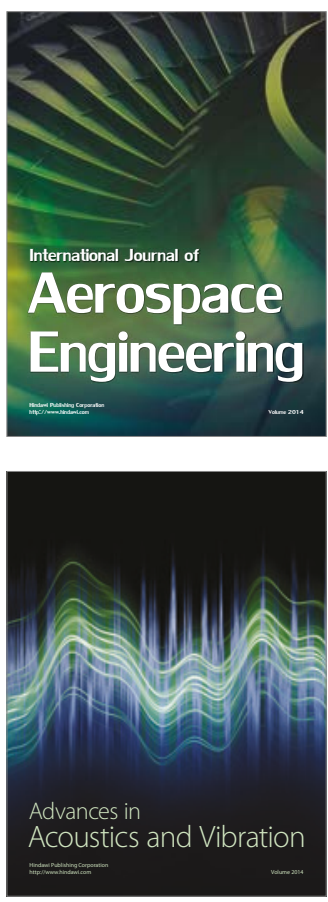

Sensor Networks 